\title{
Generation and interaction of solitons in Bose-Einstein Condensates
}

\author{
S. Burger ${ }^{1 *}$, L. D. Carr $^{2}$, P. Öhberg ${ }^{3}$, K. Sengstock ${ }^{1}$, and A. Sanpera ${ }^{4}$ \\ ${ }^{1}$ Institut für Quantenoptik, Universität Hannover, Germany \\ ${ }^{2}$ Department of Physics, University of Washington, Seattle, WA 98195-1560, USA \\ 3 School of Physics and Astronomy, University of St Andrews, North Haugh, St Andrews, Fife KY16 9SS, Scotland \\ ${ }^{4}$ Institut für Theoretische Physik, Universität Hannover, 30167 Hannover, Germany
}

Generation, interaction and detection of dark solitons in Bose-Einstein condensates is considered. In particular, we focus on the dynamics resulting from phase imprinting and density engineering. The generation of soliton pairs as well as their interaction is also considered. Finally, motivated by the recent experimental results of Cornish et al. (Phys. Rev Lett. 85, 1795, 2000), we analyze the stability of dark solitons under changes of the scattering length and thereby demonstrate a new way to detect them. Our theoretical and numerical results compare well with the existing experimental ones and provide guidance for future experiments.

\section{INTRODUCTION}

Bose-Einstein condensates (BEC's) offer a unique possibility of studying nonlinear effects using matter waves. This has been spectacularly shown in the recent BEC experiments which demonstrate, among other things, the possibility of four wave mixing [1], the creation of topological structures such as vortices [2],3], the creation of solitons [4], as well as other demonstrations of the superfluid character [7].

Solitons are one dimensional waves that propagate without spreading in a nonlinear medium. Their shape remains unaltered after interacting with other solitons. The nonlinear Schrödinger equation, which accurately describes dilute BEC's at zero temperature, supports soliton solutions for attractive as well as for repulsive 2-body interactions [8]. These solutions correspond to macroscopically excited states of the mean field of the condensate. For a single component condensate, the 3D time-dependent nonlinear Schrödinger equation, also known as the Gross-Pitaevskii equation (GPE), reads

$$
i \hbar \frac{\partial}{\partial t} \Psi(\vec{r}, t)=\left\{-\frac{\hbar^{2}}{2 m} \nabla^{2}+V(\vec{r})+g|\Psi(\vec{r}, t)|^{2}\right\} \Psi(\vec{r}, t) .
$$

Here $g=4 \pi \hbar^{2} a / m$, where $a$ corresponds to the $s$-wave scattering length for binary collisions between atoms, $m$ refers to the mass of the atoms and $V$ to the trap potential. For repulsive interactions $(a>0)$, solitons are characterized by a local density minimum together with a sharp phase gradient of the wave function at the position of the minimum. In this case, the nonlinear effective mean field potential term $g|\Psi(\vec{r}, t)|^{2}$ balances the dispersion of the wavefunction caused by the kinetic energy. Because there is a notch in the density these solutions are termed dark solitons. Only when the density of the condensate exactly vanishes at the density minimum, is the resulting soliton stationary. Such a standing soliton has exactly a phase jump of $\pi$ between the two parts of the condensate connected by it and its velocity is zero. In general, the local minimum density can range from maximal to zero depth with the associated soliton velocity, $\dot{q}$, ranging from zero to the speed of sound, $c_{s}$, in the condensate, i.e. $0 \leq \dot{q} \leq c_{s}=\sqrt{n_{0} g / m}$, where $n_{0}$ refers to the mean density in the condensate. In a homogeneous 1D condensate, a soliton solution can be analytically obtained in an elegant way by using the inverse scattering method [9]. The wavefunction corresponding to a dark soliton located at $q$ propagating along the $z$-axis with speed $v$ is described by

$$
\begin{aligned}
& \Psi_{\text {dark }}(z, t)=\sqrt{n_{0}}\left\{i \frac{v}{c_{s}}+\right. \\
& \left.\sqrt{1-\frac{v^{2}}{c_{s}^{2}}} \tanh \left[\sqrt{1-\frac{v^{2}}{c_{s}^{2}}} \frac{(z-v t)}{\sqrt{2} l_{0}}\right]\right\} e^{-i g n_{0} t / \hbar} .
\end{aligned}
$$

In the case of a dark soliton propagating along the $z$-axis in an elongated $3 \mathrm{D}$ condensate, $z$ refers to the position of the notch $(x-y)$ plane, and $v$ the constant velocity of such a plane with respect to a stationary background. The soliton size is of the order of twice the healing length $l_{0}=\hbar / \sqrt{m g n_{0}}$.

In contrast, for condensates with attractive interactions $(a<0)$ soliton solutions are characterized by a maximum in the density profile without any phase jump across it. These solitons are termed bright solitons, and the solution corresponding to a bright soliton reads:

$$
\Psi_{\text {bright }}=\sqrt{n_{0}} \operatorname{sech}\left(\frac{z-v t}{l_{0}}\right) e^{-i 2 m v z / \hbar} e^{-i g n_{0} t / \hbar}
$$

The ground state of a condensate with attractive interactions and sufficiently large nonlinearity, i.e., $g>g_{\min }$, is, in fact, a bright soliton [14].

Dark and bright solitons described by the nonlinear Schrödinger equation have been extensively studied in the context of nonlinear optics (see [10] and references therein). In condensates only dark solitons have very recently been observed as nonlinear matter waves [4]. A 
crucial difference between optical and matter wave solitons appears at first glance. While optical solitons are created in optical guides, i.e. in a cylindrical medium which is a priori unbounded, a condensate is always confined in a trap. To what extent the boundary conditions affect the properties and stability of the solitons has been the subject of recent theoretical studies (see e.g. [11]).

The literature concerning bright solitons in matter waves is not very extensive [8,12 15]. Condensates with attractive interactions in 2 and $3 \mathrm{D}$ are unstable objects that collapse very rapidly when the particle number becomes too large [16, 17]. Therefore a bright soliton in a condensate with attractive interactions is also an unstable object. However, since quasi-1D condensates with attractive interactions are stable, bright solitons could be generated in them [18]. Alternatively, an interesting approach based on vector solitons in two-component condensates (with repulsive interactions) can also be used to to study bright solitons in 2 and 3D condensates. By creating a dark soliton in one of the species one can induce a bright soliton-like structure in the other one [6, 15, 19. which fills the minimum of the first species. Since a condensate with positive scattering length is stable, the bright soliton thus created is no longer limited by the collapse of the condensate. Finally, it has been recently shown that with the well-controlled use of Feshbach resonances in ${ }^{85} \mathrm{Rb}$, it is possible to make a condensate with attractive interactions in a very controllable way [20,21. This opens a new way to study bright solitons in matter waves.

The study of matter wave solitons, both experimentally and theoretically, principally involves three different aspects: their generation, coherent evolution including coherent effects during detection, and incoherent evolution and dissipation. So far the observed solitons in one component BEC's have been generated by the method of phase imprinting. This method, originally proposed to generate vortices, has become a very efficient tool to engineer the phase in condensates [22,23]. Optimization of the phase imprinting method has been recently discussed by Carr et al. [24], where initially not only the phase but also the density is properly engineered. A proper combination of both effects in a quasi-homogeneous condensate produces a stable, standing soliton whose properties can be used to test fundamental aspects of many-body theory such as quantum and thermal fluctuations.

The coherent evolution of solitons refers to the evolution before dissipation takes place. While a condensate has a lifetime on the order of seconds, the recent experimental results have shown that for solitons the lifetime is on the order of 15 milliseconds (in one component condensates). For shorter times the Gross-Pitaevskii equation provides an accurate description of the soliton dynamics $\llbracket 6$. In homogeneous systems and in the absence of dissipation and/or thermal phonons, solitons maintain a constant velocity. In elongated harmonic traps a dark soliton (and in 3D the nodal plane of the soliton) oscil- lates in the trap with a frequency $\Omega=\omega_{a} / \sqrt{2}$ where $\omega_{a}$ corresponds to the axial trap frequency 11. Coherent evolution also concerns the generation and interaction of pairs of solitons. This coherent soliton evolution has been recently studied in the context of two-component condensates, where new and rich dynamics appears [19].

Dissipative effects include both dynamical and thermal instabilities. Dynamical instabilities are due to the fact that solitons are indeed one dimensional objects. When embedded in higher dimensions (i.e. 2 or $3 \mathrm{D}$ condensates) their stability strongly depends on the geometry of the condensate. For sufficiently elongated traps with a high transverse confinement it is not possible to excite the transverse modes of the trap and the soliton is then dynamically stable. On the contrary, for a looser transverse confinement the transverse modes can be excited, making the soliton plane bend and undergo a snake instability. As a result, the soliton decays into phonons and more stable structures such as vortices and vortex rings 66,25,26], or even more exotic objects such as svortices 27. Depending on the trap geometry, dynamical instabilities may occur on a shorter time scale than thermal instabilities. One should point out here that the dissipatory behavior of solitons corresponding to dynamical instabilities can also be described, using the GrossPitaevskii equation, as coherent but unstable evolution 28,29.

Thermal instabilities appear due to the fact that solitons are collective excited states of the mean field of the condensate, and, therefore, decay into the ground state within a finite time. The dissipation consists in scattering of phonons on the soliton's notch plane. Studies of dissipation, which are related to the interaction of a soliton with a thermal cloud, demand one to go beyond the GPE and use the Bogoliubov-deGennes equations which describe collective modes such as phonons. Since a soliton can be regarded as a particle with negative mass, such scattering accelerates the soliton until it reaches the effective sound velocity and vanishes. This scenario has been described by Fedichev et al. [28], and has been recently studied for 3D solitons in elongated traps by Muryshev et al. [30]. Both theory and experiment indicate that the life time of the soliton due to thermodynamic instability is of the order of $15 \mathrm{~ms}$. This contrasts with the lifetime of vortices which is of the order of a few seconds [2,3].

This paper focuses on the generation and coherent evolution of dark solitons in one-component condensates in elongated traps. It is organized as follows: Section II addresses the issue of the generation of solitons. In Section III the problem of soliton detection is investigated and the dynamics concerning the opening of the trap are studied. Section IV is devoted to the interaction between solitons. We discuss therein the experimental conditions under which the effects due to interactions can be observed. In the latter, we study the stability of a soliton when the scattering length of the condensate is changed in a controllable way. This study is stimulated by the recent experiments in ${ }^{85} \mathrm{Rb}$ by Cornish et al. [20,21]. Fi- 
nally we present our conclusions in Section V.

\section{GENERATION OF DARK SOLITONS}

In this section we focus on the generation of dark solitons in one component Bose-Einstein condensates by three distinct methods: (a) phase imprinting, (b) density engineering, i.e., non-adiabatic changes of the potential confining the condensate and (c) combination of the previous two methods.

As already mentioned in the introduction, even for thermodynamically unstable states such as solitons the GPE provides an excellent tool with which to describe the coherent dynamics on the relevant timescale. To avoid the effect of dynamical instabilities we restrict our analysis to the following configurations: (a) cigar-shaped condensates with a high aspect ratio and (b) quasi-1D condensates. These quasi-1D condensates can be obtained for both harmonic and box-like potentials. For the former, the radial confinement frequency is required to be much larger than the mean field interaction between particles [30]. For the latter, the healing length is required to be of the same order as the transverse box length. These quasi-1D condensates can be experimentally realized by loading a condensate from an elongated magnetic trap into a dipole trap created by a blue detuned LaguerreGaussian laser beam [31,32].

\section{A. Phase Imprinting}

The method of phase imprinting consists of passing a short off-resonant laser pulse through an appropriately designed absorption plate and impinging it on a condensate. In this way one can imprint the desired phase structure on the condensate and hence create a dark soliton 22,23.

The Gross-Pitaevskii equation reduces to an effective one-dimensional nonlinear Schrödinger equation (NLSE) when the radial frequency is larger than the mean particle interaction and when the longitudinal dimension of the confining potential is much longer than its transverse ones

$$
\begin{aligned}
i \hbar \frac{\partial}{\partial t} \Psi(z, t)= & \left\{-\frac{\hbar^{2}}{2 m} \frac{\partial^{2}}{\partial z^{2}}+V(z)+\right. \\
& \left.\tilde{V}(z, t)+g|\Psi(z, t)|^{2}\right\} \Psi(z, t) .
\end{aligned}
$$

Here $\tilde{V}(z, t)$ describes the interaction with the external laser, i.e., denotes the dipole potential generated by the far detuned laser pulse which acts only in one part of the condensate, and $V(z)$ refers to the time-independent trapping potential which remains constant during the whole process. Let us review here how the phase imprinting can lead to the creation of a soliton [22]. For a laser pulse duration shorter than the correlation time of the condensate $\tau_{c o r}=\hbar / \mu$, where $\mu$ is the chemical potential, the wave function acquires a local phase factor $e^{-i \phi}$ without changing the condensate's density profile. To generate the appropriate phase distribution which leads to a soliton, it is sufficient to use a potential which acts only on half of the condensate, e.g.

$$
\tilde{V}(z, t)=\frac{\hbar \Delta \phi}{2}\left(1+\tanh \left[\frac{z-z_{0}}{0.45 l_{e}}\right]\right) \times f(t),
$$

where $f(t)$ is the temporal envelope of the laser pulse normalized to $\int f(t) d t=1$. This potential imprints a phase

$$
\phi(z)=\frac{\Delta \phi}{2}\left(1+\tanh \left[\frac{z-z_{0}}{0.45 l_{e}}\right]\right),
$$

where $l_{e}$ refers to the width of the potential edge, which in turn determines the steepness of the imprinted phase gradient at $z_{0}$. Attainable experimental values correspond to a $10-90 \%$-absorption width of the phase step. For this reason we use a factor of 0.45 in Eq.6. In accordance to this limit, the experimental values $l_{e}$ correspond to $l_{e} \geq 2 \mu \mathrm{m}$. Thus the phase of a dark soliton is composed of two areas of constant phase connected by a steep gradient.

In an elongated cigar-shaped condensate, no matter how accurately the phase-imprinting method is implemented, it is not possible to make a standing soliton. The soliton thus generated will be a moving soliton whose speed and depth is directly related to $l_{e}$ and the amplitude of the imprinted phase $\Delta \phi$. Figure 1 shows the results of a numerical simulation for the time evolution of the density and phase of the condensate within the first millisecond after a phase with a phase gradient of $\Delta \phi=\pi$ and $l_{e}=2 \mu \mathrm{m}$ has been imprinted.

The imprinted phase profile leads to a velocity field, $v_{z}(z)=(\hbar / m) \partial \phi(z) / \partial z$. During the evolution on a timescale of the correlation time, this velocity field leads to a reduction of density in the region $\partial v_{z} / \partial z>0$ $\left(z>z_{0}\right)$, whereas in the region $\partial v_{z} / \partial z<0\left(z<z_{0}\right)$ the density increases. After the minimum and the maximum in the density have fully developed, they begin to back-react significantly, as may be seen in the evolution of the phase. The region of the phase gradient begins to change and leads to a change in the dynamics of the density distribution. The phase gradient splits up into two regions with phase gradients of similar shapes $\left(\Delta \phi_{1} \approx \Delta \phi_{2} \approx \Delta \phi / 2\right)$. The density maximum is connected with one of the phase gradients and moves approximately with the speed of sound, $c_{s}=\sqrt{4 \pi n_{0} a} \hbar / m$, towards negative $z$-values. As time increases the density wave broadens due to dispersion and to the repulsive two-particle interactions. In contrast, for the minimum propagating towards positive $z$-values, the reduced interaction energy results in a compensation of the dispersion. This leads to an increase in the steepness of the gradient together with a reduction of the width of the minimum. In this process, a second, less pronounced minimum together with additional density perturbations are created. 
As may be seen in Fig. 2, the created pronounced minimum, in connection with the tanh-phase distribution, propagates as a stable solitary wave.

The time scale needed for such a structure to develop is approximately given by $\tau_{d s} \approx \tau_{c o r} \cdot\left(l_{e} / l_{0}\right)$, where $\tau_{c o r}$ and $l_{0}$ correspond to the correlation time and healing length of the condensate, and the phase step connected with the soliton, $\Delta \Phi_{2}$, accounts for approximately one half of the initially imprinted phase step $\Delta \phi$.

The phase imprinting method depends very strongly on the width of the potential edge $l_{e}$ as well as on the value of the imprinted phase difference $\Delta \phi$. For a width much larger than the healing length, $l_{e} \gg l_{0}$, the time needed for the dark soliton to arise is significantly enlarged, and only shallow solitons can be generated. For example, for a phase width of $l_{e}=5 \mu \mathrm{m}$, but otherwise identical parameters to those used in Fig. (11), the soliton structure develops only after an evolution time of $t_{e v} \approx 15 \mathrm{~ms}$. On the other hand, a phase imprinting with phases $\Delta \Phi>\pi$ but with the same imprinting width $l_{e}$ leads to a faster development of soliton structures, accompanied always by the simultaneous creation of multiple solitons.

\section{B. Creation of dark solitons by density engineering}

The possibility of creating solitons in a BEC by engineering only the phase suggests that it should also be possible to create solitons by purely engineering the density distribution. This would be equivalent to the creation of optical dark solitons by intensity modulations of a light field propagating in a nonlinear medium [33].

Experimentally it is simple to engineer the density of a condensate. For instance, one can modify the magnetic trapping potential in which the BEC forms with an additional optical dipole potential of a far blue detuned laser beam, which is focused to form a thin "light-sheet" perpendicular to the long axis of the cigar-shaped condensate. The spot size of the focus can easily be chosen to be much smaller than the axial size of the condensate. Therefore, in the Thomas-Fermi limit, the density distribution of the BEC will be described by an inverted parabola, except for the region where the laser focus is applied. This creates a local minimum, $n_{\min }$, with a relative density, $\beta=n_{\min } / n_{0}$, which is controllable by the laser power. Here $n_{0}$ is the density at the laser focal position for negligible laser power, $P=0$. For a Gaussian laser beam the shape of the density distribution in the vicinity of the local minimum will be approximately an inverted Gaussian. The phase distribution remains constant over the whole condensate. In order to generate soliton structures the dipole potential is nonadiabatically switched off while the magnetic trap potential is kept on. The phase and density distribution of the condensate adjusts to this new potential by creating pairs of equal but counter-propagating solitons. Note that the total phase over the condensate is conserved in this process. $\beta$ should be $\gtrsim 0.01$ in order to maintain phase coherence between the two portions of the condensate on either side of the density notch.

We have numerically simulated the creation of dark solitons in a BEC in the range of parameters accessible to current experiments by pure density engineering. For instance, Figure 3 (a) shows, $10 \mathrm{~ms}$ after the non-adiabatic removal of the optical potential, the density and phase distribution of a ${ }^{23} \mathrm{Na}$ condensate with $N=5 \times 10^{6}$ atoms. In this case the magnetic trap has a radial trapping frequency $\omega_{\perp}=320 \mathrm{~Hz}$ and an aspect ratio of $\lambda=25$. On the other hand, the optical detuned laser has a Gaussian half width at $1 / e^{2}$ of $W=2 \mu \mathrm{m}$, and its intensity is assumed to be such that it corresponds to $\beta=0.9$. The density profile of the condensate shows three pairs of counter-propagating dark solitons with different velocities while the phase distribution depicts the corresponding steep phase gradients in the vicinity of the solitons.

For a wider laser focus with respect to the healing length, the number of dark soliton pairs increases; Fig. 3(b) shows the situation $10 \mathrm{~ms}$ after the switching off of a laser beam with $\beta=0.6$ and $W=12 \mu \mathrm{m}$. One sees from the figure that after this time most of the initial density deformation has already been transformed into stable density minima. For a narrower laser focus with respect to the healing length it is possible to produce a single pair of solitons 24.

\section{Phase and density engineering}

Finally, in this last subsection we briefly summarize the results which show that by a proper combination of phase and density engineering a single standing dark soliton can be created in a quasi-one dimensional BEC [24]. In this new scenario, a box like confining trap potential is used for the condensate. For a harmonic potential the Thomas Fermi radius scales as $N^{1 / 5}$ and therefore the healing length scales as $N^{-1 / 5}$, while for a box-like confinement the healing length scales as $N^{-1 / 2}$. Since the size of the soliton is of the order of twice the healing length it should be possible, for a box-like confinement with the appropriate choice of experimental parameters, to dynamically observe the generated soliton in situ, i.e., without needing to first expand the condensate. The method of density and phase engineering is simply a combination of the two above explained methods. First a density minimum is created by adiabatically ramping the intensity of a focused laser into an initially uniform BEC (see Fig 4 ). The focused laser field is abruptly switched off and a second far detuned laser pulse of uniform density is shined on one half of the condensate (phase imprinting). Thus the density minimum acquires the appropriate phase distribution. In this way one can create a single standing dark soliton. Variations on this technique allow one to create 
in a well-controlled manner asymmetric soliton pairs and various combinations of larger numbers of solitons.

\section{OPENING OF THE TRAP}

In current experiments using phase imprinting the size of the created soliton $\simeq 2 l_{0}$ is, nevertheless, smaller than the diffraction limit of the wavelength of the imaging radiation, so the soliton cannot be observed in situ [4,5]. To overcome this problem the trap is suddenly switched off, so that the condensate, and therefore the soliton, expands freely for a few milliseconds $\left(t_{T O F}\right)$ and thus becomes detectable via absorption imaging [1]. In this section we analyze the dynamics associated with the opening of the trap and the ballistic expansion. We consider a cigarshaped geometry with a large aspect ratio. The ballistic expansion then occurs principally in the transverse direction. The dynamics related to the opening of the trap is complicated, since the abrupt switching off of the trap potential modifies not only the density distribution but also the phase structure present in the condensate. Our 3D numerical simulations show that as the condensate expands the soliton velocity diminishes very rapidly while its depth increases. Simultaneously a new minimum in the density distribution appears in the vicinity of the density maximum connected with one of the phase gradients. This new density minimum observed in the experiments [4 travels opposite to the soliton direction with a velocity smaller than the sound velocity. Since a soliton can be interpreted as a particle with a negative mass, by opening the trap the soliton acquires kinetic energy and, therefore, its velocity decreases until eventually it becomes a standing soliton.

A condensate in a quasi-1D trap (infinitely long cylinder along the axial direction) admits a scaling function for the wave function [34, 35]. Following Ref. [34] we reexpress the GPE, Eq.(1), in cylindrical coordinates for the stationary case

$$
\begin{aligned}
& \left\{-\frac{\hbar^{2}}{2 m}\left(\Delta_{\rho}+\Delta_{z}\right)+\right. \\
& \left.\frac{m \omega_{\rho}^{2} \rho^{2}}{2}+g|\Psi(\rho, z)|^{2}-\mu\right\} \Psi(\rho, z)=0 .
\end{aligned}
$$

The corresponding stationary soliton solution reads

$$
\left.\Psi_{\text {stat }}=\sqrt{\frac{\mu}{g}} \sqrt{1-y^{2}} \tanh \left(z \sqrt{1-y^{2}}\right)\right) e^{-i \mu \hbar / t}
$$

where $y=\rho / R_{T F}$ and $z=z / l_{0}$. We assume here for the radial coordinate that $\mu \simeq \mu_{T F}$. Switching off the trap abruptly corresponds to making $\omega_{\rho}$ suddenly zero. A scaling solution takes the form

$$
\Psi(\rho, z, t)=\frac{1}{b(t)} \Psi_{\text {stat }}\left(\frac{\rho}{b(t)}, \frac{z}{b_{z}(t)}, \tau\right) e^{i \phi(\rho, z, t)} .
$$

By substituting Eq. (9) into Eq. (7) and splitting the real and imaginary part, a solution is found for an appropriate choice of the phase $\phi$, giving rise to the scaling $b_{z}(t)=$ $\sqrt{1+\omega_{\rho}^{2} t^{2}}$. This approach, which is valid for $\omega_{\rho}^{-1} \leq$ $t_{T O F} \leq \mu / \hbar \omega_{\rho}^{2}$, predicts a soliton velocity

$$
v(\tau)=v(0) \frac{\ln \left(\omega_{\rho} \tau+\sqrt{\omega_{\rho}^{2} \tau^{2}+1}\right)}{\omega_{\rho} \tau}
$$

where $\tau=t-t_{\text {open }}$ so that $v(0)$ corresponds to the velocity of the soliton at the time the trap is suddenly switched off $\left(t_{\text {open }}\right)$. This scaling law agrees very well with the numerical results obtained by solving the time dependent GPE, as is shown in Fig.(5), as well as with the experimental data of Ref. [4].

\section{INTERACTING SOLITONS}

Solitons propagate in a nonlinear medium without changing their shape even when they interact with each other. The interaction between optical solitons has been intensively studied in the propagation of light in monomode optical fibers (see Ref. [10] and references therein). Zakharov and Shabat demonstrated that homogeneous Bose-Einstein condensates support multi-soliton solutions and that solitons interact with each other like classical particles with a short range repulsive interaction [9]. The signature of this repulsive interaction manifests itself as a negative shift in the position of each soliton after interaction as compared to the position of a single free moving soliton. For an untrapped homogeneous 1D condensate this shift can be analytically calculated using the inverse scattering method. In this case the GrossPitaevskii equation reduces to

$$
i \frac{\partial}{\partial t} \Psi(z)=\left[-\frac{1}{2} \frac{\partial^{2}}{\partial z^{2}}+|\Psi(z)|^{2}\right] \Psi(z) .
$$

where $z$ is in units of the healing length and time in units of $\hbar / 2 g n_{0}$.

For a repulsive condensate, with the boundary conditions $|\Psi(z, t)|^{2} \rightarrow$ constant, a soliton solution moving with constant velocity through the condensate can be reexpressed as 9 , 36.

$$
\Psi(z, t)=\frac{(\lambda+i \nu)^{2}+\exp \left(2 \nu\left(z-z_{0}-\lambda t\right)\right)}{1+\exp \left(2 \nu\left(z-z_{0}-\lambda t\right)\right)}
$$

where $\lambda^{2}+\nu^{2}=1$ and $z_{0}$ is the initial position of the soliton at $t=0$. The parameter $\lambda$ characterizes the amplitude and the velocity of the soliton in units of $c_{s}$. In these units $-1 \leq \lambda \leq 1$, where $\lambda= \pm 1$ corresponds to a completely filled soliton (zero depth) moving with the speed of sound, whereas $\lambda=0$ corresponds to a standing soliton. 
To calculate the spatial shift due to a soliton-soliton interaction one simply compares each final soliton position to what it would have been had it not undergone a collision. For two solitons with velocities $\lambda_{1}$ and $\lambda_{2}$, the resulting shifts are given by [9, 36

$$
\begin{aligned}
& \delta z_{1}=\frac{1}{\nu_{1}} \log \left[\frac{\left(\lambda_{1}-\lambda_{2}\right)^{2}+\left(\nu_{1}+\nu_{2}\right)^{2}}{\left(\lambda_{1}-\lambda_{2}\right)^{2}+\left(\nu_{1}-\nu_{2}\right)^{2}}\right], \\
& \delta z_{2}=\frac{1}{\nu_{2}} \log \left[\frac{\left(\lambda_{1}-\lambda_{2}\right)^{2}+\left(\nu_{1}+\nu_{2}\right)^{2}}{\left(\lambda_{1}-\lambda_{2}\right)^{2}+\left(\nu_{1}-\nu_{2}\right)^{2}}\right] .
\end{aligned}
$$

If the solitons have equal velocities, i.e. $\lambda_{1}=-\lambda_{2}$, the shift is the same for both of them:

$$
\delta z=-\frac{\log \left[\left|\lambda_{1}\right|\right]}{\nu_{1}} .
$$

Generally speaking, to see how the inhomogeneity due to the trapping potential affects the interaction dynamics between solitons one has to turn to numerical solutions of the Gross-Pitaevskii equation. To this end, we solve the GPE when two opposite phase gradients are imprinted in a cigar-shaped condensate

$$
\phi(z)=\frac{\pi}{2}\left[\tanh \left(\left(z-z_{1}\right) / l_{e 1}\right)-\tanh \left(\left(z-z_{2}\right) / l_{e 2}\right)\right],
$$

where $z_{i}$ and $l_{e i}$ denote the positions of the phase gradients and the width of the potential edge respectively. Thus, at $t=0$ the wavefunction of the condensate reads

$$
\Psi(z, t=0)=e^{-i \phi(z)} \psi(z),
$$

where $\psi(z)$ is the ground state solution of the GrossPitaevskii equation. After a time of the order of the correlation time, such a phase distribution generates two counter propagating solitons (two notch planes located at $z_{1}$ and $\left.z_{2}\right)$ moving with velocities $\left(\lambda_{1}=-\lambda_{2}\right)$, together with two counter propagating density waves (density maxima) that move with the speed of sound. The soliton positions are monitored by following the density notch in the condensate. Figs. 6 and 7 display the results of a full 3D calculation corresponding to the experimental conditions discussed in [4], where the soliton planes are initially separated by 20 and 35 microns respectively. Due to the change in the background density of the condensate, the solitons first accelerate, and then cross each other. In Fig. 6 for the case of an initial separation of 20 microns the two solitons overlap strongly during the collision, which lasts for approximately $1 \mathrm{~ms}$. In Fig. 7 for an initial separation of 35 microns, the solitons collide with such a high velocity, that the short interaction time makes the position shifts negligible.

In spite of the $3 \mathrm{D}$ character of the elongated trap one can still use the analytical results [9] from the homogeneous $1 \mathrm{D}$ case by applying a local density approximation where the condensate density is considered constant in the region of the soliton plane. This approximation is valid away from the the edges of the trap. Inserting the parameters used in the Hannover soliton experiment [4] for ${ }^{87} \mathrm{Rb}$ with $\mathrm{N}=10^{5}, \mathrm{a}=5.7 \mathrm{~nm}$, and an initial $v_{\text {soliton }}=3.3 \mu \mathrm{m} / \mathrm{ms}$, and assuming an effective cross section area of $S=25 \mu^{2}$, the predicted shift in the position of the solitons is $\delta z=-0.1 \mu \mathrm{m}$. This calculated value is in agreement with the numerical simulations. Such a small positional shift cannot be detected experimentally. In order to obtain a shift of the order of $10 \mu \mathrm{m}$ the velocity difference should be $\left|v_{1}-v_{2}\right| \sim 10^{-6} c_{s}$.

Finally, in Fig 8 we display the results (1D) for the collision between two dark solitons created with phase gradient of $l_{e}=10$. The initial velocities are very small $\left(v \sim 0.05 c_{s}\right)$. Thus the interaction time becomes very large. The solitons are clearly seen to bounce off each other like classical particles undergoing an elastic collision. The shift in the positions is, however, still very small. Here we have chosen the density to be $4 \times 10^{13} \mathrm{~cm}^{-3}$ which again corresponds to a condensate with the effective cross sectional area $S=25 \mu \mathrm{m}^{2}$.

In order to unambiguously detect experimentally a signature of the interaction between solitons in condensates, one should create two solitons with very similar velocities propagating in the same direction. The solitons will thus interact for a long time. From Eqs. (13) and (14) it is clear that this scenario can amount to an arbitrarily large shift. This requires, however, a very long condensate. Such condensates are becoming experimentally available 32,37 in reduced geometries. On the other hand, this drawback could be removed by creating the condensate in a ring geometry. Another possibility is to create solitons in a hard wall geometry, as for example in a hollow blue-detuned laser beam with laser light sheet endcaps, so that the solitons will reflect from the ends without changing their form, since the wavefunction at the endcaps acts as a pinned soliton 38 .

\section{STABILITY OF DARK SOLITONS FOR NEGATIVE SCATTERING LENGTHS}

Recent experiments concerning the use of Feshbach resonances 21 to change both the magnitude and the sign of the scattering length of condensates with alkali atoms offers a new range of phenomena to study. In particular, a carefully controlled study of the outstanding problem of collapse of the condensate for negative scattering length becomes possible [39]. As already mentioned in the previous sections, the form of a soliton depends on the sign of the scattering length. For positive scattering lengths the stable soliton solution is a density notch, i.e, a density minimum; for sufficiently strong negative scattering lengths the stable solution is a bright soliton which is a density peak.

The possibility of changing the scattering length from positive to negative values opens the question of the stability of dark solitons in attractive condensates. Let us first reconsider the stability of dark solitons when adiabatically changing the scattering length. Since a soliton is a particular solution of the Gross-Pitaevskii equation 
for a well defined scattering length, it is reasonable to assume an adiabatic change of the scattering length will change the velocity and depth of the soliton gradually.

On the contrary, an abrupt change in the condensate's scattering length will destroy the soliton. For negative scattering lengths the physical and mathematical situation changes dramatically. If we only consider low densities and neglect three-body recombination - which becomes important at high densities and produces additional kinetic energy - in the absence of any solitons, the instability of the condensate is seen as a collapse of the condensate's wavefunction. This is shown in Fig. 9, where one can see a shrinking cloud as time proceeds and where no solitons are present. In these simulations we have used a small ${ }^{87} \mathrm{Rb}$ condensate with 5000 atoms in the same cigar-shaped trap previously discussed. We change the scattering length from its initial value $a=5.7 \mathrm{~nm}$ to $a \rightarrow-0.1 \mathrm{a}$. In the presence of a dark soliton, (Fig. 10) the scenario changes dramatically. The soliton splits the cloud into two separate parts which independently continue to collapse. A direct consequence of non-adiabatically changing the sign of the scattering length in the presence of a soliton is the creation of a large number of density waves. This effect speeds up the collapse of the wave function because of the local increase in the density. A more careful study which takes into account three-body recombination processes is needed to investigate the dynamics for longer times.

\section{CONCLUSIONS}

We have discussed the generation, evolution, and interaction of dark solitons in matter waves. We have first reviewed different approaches to generate standing or moving dark solitons in one component condensates. The interaction dynamics between dark solitons have also been addressed and we have discussed under which circumstances the interaction can be observed experimentally. We conclude that in present experiments using cigarshaped condensates with a large aspect ratio, a conclusive signature of the soliton interaction cannot be observed. However, by using other geometries, such as quasi-1D or toroidal condensates, the interaction could be unambiguously detected experimentally. A stationary wave in the form of a density notch or peak, even if it moves with less than the speed of sound, cannot truly be called a soliton until it is demonstrated that it interacts as one, since that is the defining characteristic which gives solitons their particle-like nature.

Finally, we have discussed the stability of dark solitons to sudden changes of the sign and value of the scattering length. We find that the presence of a dark soliton can be unambiguously detected by the radical change in the dynamics of a collapsing cloud when a Feshbach resonance is used to tune the scattering length negative: the cloud splits in two.

\section{ACKNOWLEDGMENTS}

We acknowledge support from SFB407 of the Deutsche Forschungsgemeinschaft, the National Science Foundation Grant CHE97-32919, PESC BEC2000+ and EPSRC. Discussions with K. Bongs, M. Lewenstein, L. Santos, J. Brand, W. Ertmer and in particular G. Shlyapnikov (sec III) are gratefully acknowledged.

[1] L. Deng, E.W. Hagley, J. Wen, M. Trippenbach, Y. Band, P.S. Julienne, J.E. Simsarian, K. Helmerson, S.L. Rolston, and W.D. Phillips, Nature 398, 218 (1999).

[2] M. R. Matthews, B.P. Anderson, P. C. Haljan, D. S. Hall, C. E. Wieman, and E. A. Cornell, Phys. Rev. Lett. 83, 2498 (1999).

[3] K. W. Madison, F. Chevy, W. Wohlleben, and J. Dalibard, Phys. Rev. Lett. 84, 806 (2000).

[4] S. Burger, K. Bongs, S. Dettmer, W. Ertmer, K. Sengstock, A. Sanpera, G.V. Shlyapnikov, and M. Lewenstein Phys. Rev. Lett. 83, 5198 (1999).

[5] J. Denschlag, J. E. Simsarian, D. L. Feder, C. W. Clark, L. A. Colliins, J. Cubizolles, L. Deng, E. W. Hagley, K. Helmerson, W. P. Reinhardt, S. L. Rolston, B. I. Schneider, and W. D. Phillips, Science 287, 97 (2000).

[6] B.P Anderson, P.C. Haljan, C. A. Regal, D. L. Feder, L. A. Collins, C. W. Clark, E. A. Cornell, Phys. Rev. Lett. 86, 2926 (2001).

[7] O. M. Marago, S. A. Hopkins, J. Arlt, E. Hodby, G. Hechenblaikner and C.J. Foot, Phys. Rev. Lett. 84, 2056 (2000).

[8] V. E. Zakharov and A. B. Shabat, Sov. Phys. JETP 34, 62 (1972).

[9] V. E. Zakharov and A. B. Shabat, Sov. Phys. JETP 37, 823 (1973).

[10] See e.g. Y. S. Kivshar and B. Luther-Davies, Phys. Rep. 298, 81 (1998).

[11] Th. Busch, and J. R. Anglin, Phys. Rev. Lett. 84, 2298 (2000).

[12] G. Lenz, P. Meystre,and E.W. Wright, Phys. Rev. A 50, 1681 (1994).

[13] O. Zobay, S. Pötting, P. Meystre, and E.M. Wright, Phys. Rev. A 59, 643, 1999.

[14] L. D. Carr, W. P. Reinhardt, and C. W. Clark, Phys. Rev. A 62, 063611 (2000).

[15] T. Busch and R. J. Anglin, cond-mat/0012354; T. Busch "Theoretical Studies of Degenerate Inhomogenous Atomic Gases", Dissertation Thesis, Innsbruck, 2000.

[16] C.C. Bradley, C.A. Sackett, and R. G. Hulete, Phys. Rev. Lett. 78, 985 (1997).

[17] P. A. Ruprecht, M. J. Holland, K. Burnett, and M. Edwards, Phys. Rev. A 51, 4704 (1995); M. Houbiers and H. T. C. Stoof, Phys. Rev. A 54, 5055 (1996); Y. Kagan, A. E. Muryshev and G. V. Shlyapnikov, Phys. Rev. Lett. 81, 933 (1998). 
[18] L. D. Carr, M. A. Leung and W. P. Reinhardt, J. Phys. B 33, 3983 (2000).

[19] P. Öhberg and L. Santos, Phys. Rev. Lett. 86, 2918 (2001); P. Öhberg and L. Santos, to appear in J. Phys. B (2001).

[20] S. L. Cornish, N. R. Claussen, J. L. Roberts, E. A. Cornell, and C. E. Wieman, Phys. Rev. Lett 85, 1795 (2000).

[21] J.L. Roberts, N.R. Claussen, S.L. Cornish, E.A. Donley, E.A. Cornell, and C.E. Wieman, Phys. Rev. Lett 86, 4211 (2001).

[22] Ł. Dobrek, M. Gajda, M. Lewenstein, K. Sengstock, G. Birkl, and W. Ertmer, Phys. Rev. A 60, R3381 (1999).

[23] G. Andrelczyk, M. Brewczyk, E. Dobrek, M. Gajda, M. Lewenstein, submitted to Phys. Rev. A, (2001).

[24] L. Carr, J. Brand, S. Burger and A. Sanpera, Phys. Rev. A 63, 051601 (2001).

[25] R.J. Donnelly "Quantized vortices in Helium II", Cambridge University Press, 1991.

[26] J. Martikainen, K.A. Suominen, L. Santos, T. Shultz and A. Sanpera, cond-mat/0005136.

[27] J. Brand and W. P. Reinhardt, J. Phys. B 34 L113 (2001).

[28] P. O. Fedichev, A. E. Muryshev, G. V. Shlyapnikov, Phys. Rev. A 60, 3220 (1999).

[29] A. E. Muryshev, H. B. van den Linden van den Heuvell, and G. V. Shlyapnikov, Phys. Rev. A 60, R2665 (1999).

[30] A. Muryshev, G. V. Shlyapnikov, and M. Lewenstein, preprint.

[31] K. Bongs, S. Burger, S. Dettmer, D. Hellweg, J. Arlt, W. Ertmer, and K. Sengstock, cond-mat/0007381.

[32] S. Dettmer, D. Hellweg, P. Ryytty,J. Arlt, W. Ertmer, K. Sengstock, D. S. Petrov, G. Shlyapnikov, H. Kreutzman, L. Santos, and M. Lewenstein, Phys. Rev. Lett. 87, 160406 (2001).

[33] S. A. Gredeskul and Yu. S. Kivshar, Phys. Rev. Lett 62, 977 (1989); S. A. Gredeskul, Y. S. Kivshar and M. V. Yanovskaya, Phys. Rev. A 41,3994 (1990); D. Krökel, N. J. Halas, G. Giuliani and D. Grischkowsky, Phys. Rev. Lett. 60, 29 (1988).

[34] Y. Kagan, E. L. Surkov, and G.V: Shlyapnikov, Phys. Rev. A54, R1753 (1996); 55, R18 (1997).

[35] Y. Castin and R. Dum, Phys. Rev. Lett. 77, 5315 (1996).

[36] Elements of soliton theory, G.L. Lamb Jr., Wiley (1980); P. G. Drazin and R. S. Johnson, Solitons: an Introduction, (Cambridge Univ. Press, Cambridge 1989).

[37] A. Gorlitz, J.M. Vogels, A.E. Leanhardt, C. Raman, T.L. Gustavson, J.R. Abo-Shaeer, A.P. Chikkatur, S. Gupta, S. Inouye, T.P. Rosenband, and W. Ketterle, Phys. Rev. Lett. 87, 130402 (2001).

[38] W. P. Reinhardt and C. W. Clark, J. Phys. B. 30, L785 (1997).

[39] J. L. Roberts, N. R. Claussen, S. L. Cornish, E. A. Donley, E. A. Cornell, and C. E. Wieman e-print condmat/0102116 (2001).
FIG. 1. Numerical simulations (1D) showing the time evolution of the density profile of $\mathrm{a}^{87} \mathrm{Rb}$ condensate with $N=10^{5}$. The static trap has a frequency $\omega_{z}=2 \pi \times 14$ Hz. The density profile $n(z)$ and the phase are depicted respectively in (a) and (b) within the first millisecond after a phase-imprint with $\Delta \phi=\pi$ and $l_{e}=2 \mu \mathrm{m}$ has been implemented.

FIG. 2. Time evolution of the density profile $n(z)$ and the phase $\phi(z)$ for the first 5 milliseconds after a $\pi$-phase has

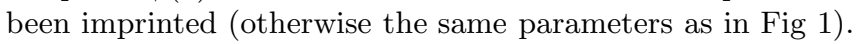

FIG. 3. Evolution of pairs of counter propagating dark solitons created by a non-adiabatic change of the BEC trapping potential. The parameters here correspond to a Na-condensate with $N=5 \times 10^{6}$ atoms in a magnetic trap of the clover-leaf type with a radial trapping frequency of $\omega_{\perp}=320 \mathrm{~Hz}$ and an aspect ratio of $\lambda=25$ : (a) $W=2 \mu \mathrm{m}$, $\beta=0.9$ and (b) $\beta=0.6$ and $W=12 \mu \mathrm{m}$. In the same figure, density profile and phase gradients are depicted. Note that the phase in (b) has been plotted modulo $2 \pi$.

FIG. 4. (a) A combination of a box-like potential and a tightly focused, blue-detuned laser beam is used to engineer the density. (b) The resulting wavefunction is phase engineered with a second, far-detuned laser beam, resulting in an initial state that resembles very much a standing dark soliton.

FIG. 5. Velocity of the soliton outside the trap versus observation time. The soliton is imprinted at $t=0$ and the trap is suddenly removed after $t=4 \mathrm{~ms}$. The curve shows the analytical scaling law, and the dots correspond to the values of the soliton velocity obtained from numerical $3 \mathrm{D}$ simulations.

FIG. 6. Interaction between solitons in a cigar shaped condensate. The solitons are initially created by the method of phase imprinting with an initial separation of 20 microns. Here $\mathrm{N}=10^{5}$ and $\mathrm{a}=5.7 \mathrm{~nm}$.

FIG. 7. The same parameters as in Fig. 6, with the solitons initially separated by 35 microns. At the time of the collision their velocities are now higher, hence the interaction time shorter.

FIG. 8. The interaction between solitons in the 1D case. The density waves created by the phase-imprinting are moving with the speed of sound and are reflected at the condensate boundary (not shown in the figure) which eventually results in crossing waves at $t=12 \mathrm{~ms}$. 
FIG. 9. The scattering length is changed at $t=2 \mathrm{~ms}$ from $a$ to $-0.1 a$. The cloud, represented here as the integrated density as a function of $z$, is starting to collapse at the onset of the negative scattering length.

FIG. 10. Same situation as in Fig. 9 with the presence of a dark soliton. The soliton splits the cloud into two parts which independently start to collapse. 
This figure "figure1.jpg" is available in "jpg" format from: http://arxiv.org/ps/cond-mat/0201155v1 
This figure "figure2.jpg" is available in "jpg" format from: http://arxiv.org/ps/cond-mat/0201155v1 
This figure "figure3.jpg" is available in "jpg" format from: http://arxiv.org/ps/cond-mat/0201155v1 
This figure "figure4.jpg" is available in "jpg" format from: http://arxiv.org/ps/cond-mat/0201155v1 
This figure "figure5.jpg" is available in "jpg" format from: http://arxiv.org/ps/cond-mat/0201155v1 
This figure "figure6.jpg" is available in "jpg" format from: http://arxiv.org/ps/cond-mat/0201155v1 
This figure "figure7.jpg" is available in "jpg" format from: http://arxiv.org/ps/cond-mat/0201155v1 
This figure "figure8.jpg" is available in "jpg" format from: http://arxiv.org/ps/cond-mat/0201155v1 
This figure "figure9.jpg" is available in "jpg" format from: http://arxiv.org/ps/cond-mat/0201155v1 
This figure "figure10.jpg" is available in "jpg" format from: http://arxiv.org/ps/cond-mat/0201155v1 